\section{Individualized risk assessment for prostate cancer}

A multi-institutional team of researchers from the US has used data from the Prostate Cancer Prevention Trial (PCPT) to develop an online prostate cancer risk calculator (available at http://www.compass.fhcrc.org/edrnnci/bin/ calculator/main.asp).

The PCPT was a phase III, randomized, double-blinded, 7-year trial that compared finasteride with placebo for the prevention of prostate cancer. To develop the risk calculator, the authors analyzed data from the placebo group, which comprised 5,519 men with a negative digital rectal examination (DRE) result and a prostate specific antigen (PSA) level of $3 \mathrm{ng} / \mathrm{ml}$ or below. Results from prostate biopsies and annual DREs and PSA tests were combined in a multivariate analysis with information on risk factors such as race, family history and age, to model the risk of prostate cancer.

In this comparatively low-risk group of men with a mean PSA level of $1.5 \mathrm{ng} / \mathrm{ml}, 21.9 \%$ developed prostate cancer and $4.7 \%$ had highgrade disease. Increased PSA level, positive family history, and abnormal DRE result were found to be independently significantly associated with an increased risk of prostate cancer. Increased PSA level, abnormal DRE, older age, and African American ethnicity were independently related to increased risk of high-grade disease. The risk of both prostate cancer and high-grade disease was reduced in patients with a negative previous biopsy. The predictive model developed by Thompson et al. tailors risk assessment to the individual patient, taking into account the fact that the risk of prostate cancer increases with rising PSA, that there is no PSA level below which men are safe from the disease, and recognizing other factors contributing to the disease.

Original article Thompson IM et al. (2006) Assessing prostate cancer risk: results from the Prostate Cancer Prevention Trial. J Natl Cancer Inst 98: 529-534

\section{Can hypermethylated Wnt- antagonist genes act as biomarkers for bladder cancer?}

Dysfunction of Wnt-antagonist genes can contribute to carcinogenesis, and aberrant promoter hypermethylation of these genes has been found in bladder tumors. Researchers have investigated whether methylation analysis of Wnt antagonists could be used to diagnose bladder cancer.

In total, 54 samples of bladder tumor tissue, and corresponding normal bladder mucosa, were analyzed for methylation status and expression levels of six Wnt-antagonist genes: SFRP1, SFRP2, SFRP4, SFRP5, WIF1, and $D K K 3$. Methylation levels of these genes were higher, and mRNA expression levels lower, in bladder tumor samples than in normal bladder mucosa. Methylation levels of SFRP2 and $D K K 3$ were significant independent predictors of bladder cancer $(P<0.05$ and $P<0.01)$; SFRP1, SFRP5 and WIF1 showed a trend towards significance. All patients with bladder cancer had hypermethylation of at least one of the six genes.

The $\mathrm{M}$ score, a new method developed for analysis of methylation of multiple genes, was used to increase the sensitivity and specificity of bladder tumor detection. M scores were calculated using hazard ratio coefficients for bladder tumor diagnosis for each gene except SFRP4. M scores were significantly higher in bladder tumors than in normal bladder mucosa $(P<0.05)$, and showed $77.2 \%$ sensitivity and $66.7 \%$ specificity for distinguishing bladder tumor from normal bladder mucosa. DNA from urine samples and from tumor tissue taken from patients with bladder tumors showed a high percentage of identical methylation; most urine DNA from healthy individuals showed no aberrant hypermethylation.

The authors conclude that $\mathrm{M}$-score analysis of Wnt-antagonist genes might be useful for noninvasive detection of bladder tumors from cellular DNA extracted from urine samples.

Original article Urakami S et al. (2006) Combination analysis of hypermethylated Wnt-antagonist family genes as a novel epigenetic biomarker panel for bladder cancer detection. Clin Cancer Res 12: 2109-2116

\section{How can we avoid the prescription of contraindicated drugs?}

Adverse events caused by prescribing drugs in violation of a 'black box' warning are thankfully rare-occurring in $<1 \%$ of those who receive a contraindicated drug, according to results of an observational study of records from 51 